\title{
Fatores econômicos e diferenças de gênero na prevalência do tabagismo em adultos
}

\author{
Economic factors and gender differences \\ in the prevalence of smoking among adults
}

Programa de PósGraduação em Economia, Centro de Ciências Sociais Aplicadas, Universidade Federal de Pernambuco. Centro de Ciências Sociais Aplicadas/Departamento de Economia Sala 102, Cidade Universitária. 50670-901 Recife PE Brasil. nlpaes@yahoo.com.br

\begin{abstract}
This article presents a study that seeks to identify the relevant economic variables in the prevalence of smoking in a group of 37 countries. The chosen methodology was to estimate multiple linear regression using the least square approach. The econometric exercise is performed by gender, seeking to examine whether there are different motivations for cigarette smoking among the adult population of men and women. The results show that although taxation is a common element in the decision of both sexes, the decision to smoke among women is also sensitive to price and other social and cultural factors. These factors were based on the fact that women who live in countries that are part of the Organization for Economic Cooperation and Development reveal a significantly higher prevalence of cigarette consumption. The evidence presented in this study, therefore, reinforces the perception that taxation is in fact a crucial tool in the control of smoking, but in the specific case of women, higher prices and the promotion of greater equality with men, are also important.
\end{abstract}

Key words Prevalence, Smoking, Gender identity
Resumo Este artigo apresenta um estudo em que busca identificar as variáveis econômicas relevantes na prevalência do cigarro para um grupo de 37 países. Utilizou-se a metodologia de estimação por regressão linear múltipla pelo método dos mínimos quadrados. O exercício econométrico é realizado por gênero, procurando examinar se há motivações diferentes para o consumo de cigarros entre a população adulta de homens e mulheres. Os resultados apontam que, embora a tributação seja um elemento comum na decisão de ambos os sexos, a decisão de fumar das mulheres é também sensivel a preços e a outros fatores sociais e culturais. Estes fatores foram apreendidos no fato de que mulheres que residem em países que fazem parte da Organização para Cooperação e Desenvolvimento Econômico apresentam, de forma significativa, maior prevalência no consumo de cigarros. As evidências apresentadas neste estudo reforçam, portanto, a percepção de que a tributação é de fato um instrumento fundamental no controle do consumo de cigarros, mas que no caso específico das mulheres, preços mais elevados e a promoção de maior igualdade com os homens, são igualmente importantes.

Palavras-chave Prevalência, Tabagismo, Identidade de gênero 


\section{Introdução}

O consumo de cigarros tornou-se corriqueiro em vários países do mundo. Dados da Organização Mundial da Saúde ${ }^{1}$ apontam que em mais de 27 países, acima de $30 \%$ da população adulta consome cigarros regularmente. Considerando somente a população masculina, em 93 países mais de $30 \%$ dos homens fumam. Longe de ser apenas um fenômeno social, a decisão de fumar também tem relação com variáveis econômicas.

O consumo de cigarros pelo mundo mais do que triplicou no período entre 1950 e 2009, passando de 1,69 trilhões para 5,88 trilhões de unidades. Porém, o comportamento da prevalência de cigarros após um pico na década de 1970 nos países desenvolvidos, começou a declinar a partir de 1980, o que resultou na diminuição do percentual da população adulta que consome cigarros nestes países. Na Europa Ocidental a redução média foi de $26 \%$ entre 1990 e 2009 . Por outro lado, nos países em desenvolvimento, a evolução da prevalência tem aumentado incessantemente. No Oriente Médio e na África, o consumo de cigarros aumentou 57\% entre 1990 e 2009. Estima-se que $38 \%$ do consumo total de cigarros em 2009 ocorreu na China ${ }^{2}$.

Há, porém, diferenças relevantes entre a prevalência de homens e mulheres. Estima-se que a prevalência entre os homens seja de cerca de dez vezes maior do que entre as mulheres ${ }^{2}$. Os mesmos autores relatam, entretanto, que este é o grupo em que o consumo de cigarros mais avança principalmente nos países em desenvolvimento.

No Brasil, os dados mostram uma clara tendência de redução da prevalência dos cigarros entre adultos. Dados apontam que em 1989, quase $32 \%$ da população de 15 anos ou mais era fumante ${ }^{3}$, percentual que se reduziu para $22,4 \% \mathrm{em}$ $2003^{4}$ e para $17,2 \%$ em $2008^{5}$. O padrão de redução foi similar para homens e mulheres. A queda para os homens foi de $43,3 \%$ para $27,1 \%$ entre 1989 e 2003 e de $27,0 \%$ para $18,4 \%$ no mesmo período para as mulheres. A maior parte da redução ocorreu durante a década de 1990 na qual o governo brasileiro promoveu forte aumento no Imposto sobre Produtos Industrializados (IPI) como forma de desencorajar o consumo de cigarros ${ }^{6}$.

Este fato realça uma característica crucial na redução do fumo entre adultos. O impacto de medidas econômicas sobre o tabagismo. Por exemplo, a Organização Mundial da Saúde tem afirmado reiteradas vezes que a tributação é a forma mais eficaz de se reduzir o uso do tabaco e encorajar o abandono do cigarro.
Este estudo procura investigar em detalhes qual a relevância de diversas variáveis econômicas e sociais na prevalência do consumo de cigarros em adultos. Como um segundo objetivo, busca-se averiguar se os impactos destas variáveis são diferentes de acordo com o gênero. Com o auxílio de técnicas econométricas, foi possível examinar, para um conjunto de 37 países, se há motivações diferentes para o consumo de cigarros entre a população adulta de homens e mulheres.

A prevalência de cigarros entre adultos é relativamente bem documentada na literatura médica. São diversos trabalhos que buscam descrever a real situação do problema e quais os principais fatores que o afetam. A estratégia tem sido basicamente a coleta e a análise de dados por meio da realização de pesquisas de campo. A contribuição deste artigo é a de aplicar uma técnica estatística, muito presente na literatura econômica para a análise dos dados socioeconômicos de um grupo de países, buscando identificar quais as características relevantes que ajudam a explicar a prevalência na população adulta e se há diferenças entre os países. Ademais, a base de dados utilizada é extensa e advém de fontes internacionais, o que permite uma análise mais ampla do que aquela que é normalmente realizada por uma pesquisa de campo.

Os resultados apontam que, embora a tributação seja um elemento comum na decisão de ambos os sexos, a decisão de fumar das mulheres é também sensível a preços e a outros fatores sociais e culturais. Estes fatores foram apreendidos no fato de que mulheres que residem em países que fazem parte da Organização para Cooperação e Desenvolvimento Econômico (OCDE) apresentam, de forma significativa, maior prevalência no consumo de cigarros.

\section{Metodologia}

A estratégia de averiguar o comportamento de homens e mulheres em relação à prevalência já foi usada por pesquisas anteriores ${ }^{7,8}$. Em ambos os estudos, o comportamento de adultos, homens e mulheres, em relação ao cigarro apresentava diferenças.

Um dos métodos possíveis de serem aplicados neste artigo é o econométrico. A econometria nada mais é do que o uso de ferramentas estatísticas para se buscar estabelecer relações entre variáveis. Em particular, será utilizada a ferramenta conhecida como regressão pelo mé- 
todo dos mínimos quadrados. Tecnicamente, este método busca o melhor ajuste para um conjunto de dados, de forma a minimizar a diferença entre os valores estimados e os dados observados. Esta técnica tem sido usada na literatura sobre tabagismo, como no estudo que concluiu que variações genéticas podem afetar a influência da tributação sobre a prevalência de cigarros ${ }^{9}$.

No presente estudo, para a avaliação dos determinantes da prevalência de cigarros foi construído o seguinte modelo econométrico:

Prev $_{i}=a+b_{1} \cdot X_{i}+b_{2} \cdot Z_{i}+e_{i}$

Onde Prev ${ }_{i}$ é a prevalência de cigarros no país $i$ que neste estudo será analisada em separado para homens, mulheres e total da população, $X_{i}$ é a matriz de variáveis independentes para o país $i$ que inclui o logaritmo do PIB per capita, o logaritmo do salário mínimo, o preço, a alíquota do imposto e a capacidade de compra de cigarros, enquanto $Z_{i}$ são as variáveis de controle não relacionadas diretamente a variáveis econômicas, como se o país $i$ faz parte da OCDE ou se a sua população é majoritariamente muçulmana, a taxa de alfabetização e a urbanização. O Quadro 1 descreve as variáveis que foram utilizadas neste estudo.

As variáveis escolhidas para o modelo de regressão buscam captar principalmente o impacto de indicadores econômicos básicos sobre a decisão de fumar. Neste sentido é que foram inseridas as variáveis preço, impostos, renda e capacidade de compra. A renda foi colocada de duas formas distintas, como per capita e pelo salário mínimo. O motivo é tentar verificar não só se a renda média de um país afeta a decisão de fumar, mas também se o rendimento esperado de grupos mais pobres também impacta nesta decisão. A capacidade de compra de cigarros foi incluída como uma maneira de se aferir se a quantidade de cigarros que pode ser comprada com a renda

Quadro 1. Descrição das variáveis utilizadas no estudo.

\begin{tabular}{|c|c|c|}
\hline Variável & Descrição & Base de Dados \\
\hline Prev & $\begin{array}{l}\text { Pessoas que estão fumando no momento. Discriminada em prevalência } \\
\text { para homens, mulheres e total da população. }\end{array}$ & OMS \\
\hline PUS & $\begin{array}{l}\text { Preço do maço de cigarros da marca mais vendida com } 20 \text { unidades em } \\
\text { US\$ a taxa de câmbio oficial. }\end{array}$ & OMS \\
\hline TAX & $\begin{array}{l}\text { Alíquota tributária total. Incluem impostos específicos, impostos sobre } \\
\text { valor adicionado, impostos sobre vendas, taxas de importações e outros } \\
\text { tributos. }\end{array}$ & OMS \\
\hline $\ln \mathrm{PIB}$ & Logaritmo do PIB per capita em US\$ a taxa de câmbio oficial. & $\begin{array}{l}\text { Heston, Summers e } \\
\text { Aten (2012). }\end{array}$ \\
\hline $\operatorname{lnSM}$ & Logaritmo do Salário Mínimo em US\$ a taxa de câmbio oficial. & OIT \\
\hline AffPIB & Logaritmo da divisão do PIB per capita pelo preço do maço de cigarros. & OIT e OMS \\
\hline MUS & $\begin{array}{l}\text { Variável dummy que indica se o país é majoritariamente muçulmano } \\
\text { ou não. }\end{array}$ & $\begin{array}{l}\text { Pew Research } \\
\text { Center }\end{array}$ \\
\hline OECD & $\begin{array}{l}\text { Variável dummy que indica se o país faz parte da Organização para } \\
\text { Cooperação e Desenvolvimento Econômico (OECD). }\end{array}$ & OCDE \\
\hline $\begin{array}{l}\text { ALFH, ALFM, } \\
\text { ALFT }\end{array}$ & $\begin{array}{l}\text { Taxa de alfabetização de adultos para homens, mulheres e população } \\
\text { total. }\end{array}$ & Banco Mundial \\
\hline URB & Taxa de urbanização. & $\begin{array}{l}\text { Índice de } \\
\text { Desenvolvimento } \\
\text { Humano (IDH) }\end{array}$ \\
\hline
\end{tabular}

Fonte: elaboração dos autores. 
média do país afeta a sua prevalência no consumo de cigarros.

As variáveis de controle tentam captar informações levantadas pela literatura. Neste sentido se incluiu no modelo as variáveis dummy OECD, para se verificar se o fato do país ser rico afeta a decisão de fumar, e MUS, que indica se o país é majoritariamente muçulmano. Também foram colocadas variáveis relacionadas ao nível educacional, como a taxa de alfabetização, que aparece com frequência nos estudos sobre prevalência, bem como a taxa de urbanização, que pode ser uma variável importante, principalmente entre as mulheres ${ }^{10}$.

A escolha das variáveis apresenta também limitações importantes. Ao se considerar apenas o preço da marca mais vendida de cigarros, perdese em representatividade, já que os preços de outras marcas não são considerados, e tampouco o preço de cigarros ilegais ou contrabandeados são incluídos na regressão. Uma segunda limitação diz respeito aos impostos, que são aqueles que incidem somente na marca mais vendida, deixando de fora os cigarros ilegais que não são tributados. A questão é que não há dados de fontes internacionais com os preços de cigarros contrabandeados para ser usado no modelo econométrico.

Os dados foram retirados de bases da Organização Mundial da Saúde ${ }^{1}$, que fornece informações sobre a prevalência entre homens, mulheres e total da população, bem como sobre alguns dos principais fatores que a afetam, como o preço do maço de cigarros de 20 unidades e o total de impostos indiretos que incidem sobre o maço. Dados sobre o PIB per capita foram retirados da Penn World Table 7.1 $1^{11}$, enquanto que informações sobre o salário mínimo saíram do global wage database da Organização Internacional do Trabalho (OIT). A informação se o país faz parte ou não da Organização para Cooperação e Desenvolvimento Econômico (OCDE) foi retirada da mesma. Quanto a religião, considerou-se predominantemente muçulmano os países na qual o percentual da população que segue esta religião é maior do que $30 \%{ }^{12}$. Por fim, as informações sobre taxa de alfabetização foram extraídas do sítio da base de dados do Banco Mundial e da taxa de urbanização do sítio do Índice de Desenvolvimento Humano (IDH) da Organização das Nações Unidas.

Somente 37 países apresentam este conjunto de dados para o mesmo ano e se constituem na amostra a ser utilizada neste estudo: Afeganistão, Argélia, Austrália, Áustria, Azerbaijão, Bangladesh, Bélgica, Benin, Bolívia, Brasil, Bulgária,
Chade, Chile, Chipre, Cingapura, Colômbia, Costa Rica, Dinamarca, Equador, Estônia, França, Gâmbia, Gana, Geórgia, Grécia, Laos, Letônia, Líbano, Lituânia, Malta, Montenegro, Nigéria, Omã, Quênia, Peru, Ruanda e Vietnã.

\section{Resultados e discussão}

A Tabela 1 apresenta as principais estatísticas descritivas para os países selecionados.

Os dados indicam que a prevalência no consumo de cigarros é bem superior para os homens do que para as mulheres. Enquanto que na média da amostra $32,87 \%$ dos homens fumam, o percentual para as mulheres é de apenas $14,05 \%$. A proporção de homens que usam cigarros é mais de duas vezes maior do que a de mulheres.

Verifica-se também forte dispersão nas variáveis preço e impostos. Dentro da amostra, os preços para um maço de cigarros variam entre US\$ 0,22 (Afeganistão) e US\$ 10,77 (Austrália), enquanto que a tributação vai de 4\% (Afeganistão) até $86 \%$ (Grécia). As variáveis de renda também apresentam grande variação, com o PIB per capita anual variando entre US\$ 519 (Ruanda) e US\$ 56.485 (Dinamarca).

Destaca-se também o fato de que a taxa de alfabetização dos homens é, em média, apenas um pouco superior ao das mulheres. O Afeganistão é o país com menor taxa de alfabetização masculina, $43,1 \%$, e também com menor taxa de alfabetização feminina, de apenas $12,6 \%$.

A taxa de urbanização, que na média é de $62,73 \%$, também apresenta uma grande dispersão, com o menor valor de 18,8\% (Ruanda) e o maior valor de $100 \%$ (Cingapura).

O modelo econométrico foi estimado pela regressão por mínimos quadrados. Os resultados serão apresentados nas Tabelas 2 a 4, começando pelo estudo da regressão para o total da população.

A tributação tem peso relevante na prevalência de cigarros. Independente do modelo, países com tributação sobre cigarros mais elevada acabam por apresentar maior prevalência. Não se trata de um resultado intuitivo. Seria razoável esperar que países com alíquotas tributárias mais elevadas apresentassem menor número de fumantes. Porém, a literatura sobre os efeitos da tributação sobre os cigarros mostra que, na verdade, a causalidade é inversa.

Como forma de combater a alta prevalência de cigarros, os países têm apelado para o aumento da carga tributária. A literatura econômica consagra a tributação como um dos principais 


\begin{tabular}{lcccrr}
\hline \multicolumn{1}{c}{ Tabela 1. Estatística descritiva. } \\
\multicolumn{1}{c}{ Variável } & Observações & Média & Desvio-Padrão & Mínimo & Máximo \\
\hline PrevH & 37 & 0,3283 & 0,1326 & 0,08 & 0,56 \\
PrevM & 37 & 0,1405 & 0,1260 & 0 & 0,38 \\
PrevT & 37 & 0,2370 & 0,1067 & 0,04 & 0,41 \\
PUS & 37 & 2,5486 & 2,5098 & 0,22 & 10,77 \\
TAX & 37 & 0,5578 & 0,2233 & 0,04 & 0,86 \\
lnPIB & 37 & 8,6318 & 1,5333 & 6,2519 & 10,9417 \\
lnSM & 31 & 7,5988 & 1,3865 & 4,8757 & 10,19 \\
AffPIB & 37 & 8,1142 & 0,8766 & 6,4144 & 9,6137 \\
MUS & 37 & 0,2432 & 0,4349 & 0 & 1 \\
OECD & 37 & 0,1891 & 0,3970 & 0 & 1 \\
ALFH & 37 & 0,8805 & 0,1621 & 0,431 & 1 \\
ALFM & 37 & 0,8156 & 0,2406 & 0,126 & 1 \\
ALFT & 37 & 0,8481 & 0,2007 & 0,281 & 1 \\
URB & 37 & 0,6273 & 0,2292 & 0,188 & 1 \\
\hline
\end{tabular}

Fonte: elaboração dos autores.

Tabela 2. Prevalência do consumo de cigarros, total da população.

\begin{tabular}{lcccr}
\hline \multicolumn{1}{c}{ Variável } & Modelo 1 & Modelo 2 & Modelo 3 & Modelo 4 \\
\hline PUS & -0.0239 & -0.0240 & -0.0214 & -0.0211 \\
& $(0.153)$ & $(0.140)$ & $(0.131)$ & $(0.128)$ \\
TAX & $0.362^{* *}$ & $0.364^{* *}$ & $0.352^{* *}$ & $0.366^{* *}$ \\
& $(0.005)$ & $(0.003)$ & $(0.003)$ & $(0.001)$ \\
lnPIB & 0.0490 & 0.0462 & 0.0101 & 0.0077 \\
& $(0.349)$ & $(0.357)$ & $(0.809)$ & -0.0142 \\
AffPIBM & -0.0762 & -0.0791 & -0.0258 & $(0.766)$ \\
& $(0.229)$ & $(0.196)$ & $(0.635)$ & - \\
lnSM & -0.0104 & -0.0116 & - & - \\
& $(0.620)$ & $(0.566)$ & - & \\
URB & -0.0446 & - & & 0.0813 \\
& $(0.730)$ & & 0.0846 & - \\
OECD & 0.0881 & 0.0884 & $(0.074)$ & - \\
& $(0.151)$ & $(0.138)$ & 0.0261 & 0.129 \\
MUS & 0.0580 & 0.0577 & $(0.632)$ & $(0.351)$ \\
& $(0.318)$ & $(0.306)$ & 0.197 & 0.00235 \\
ALFT & 0.232 & 0.230 & $(0.327)$ & $(0.988)$ \\
& $(0.276)$ & $(0.268)$ & 0.0208 & 30 \\
_cons & 0.0159 & 0.189 & $(0.901)$ & 0.637 \\
& $(0.451)$ & $(0.316)$ & 30 & 6.718 \\
N & 26 & 26 & 0.641 & 0.0126 \\
R2 & 0.703 & 0.700 & 5.601 & \\
F & 4.199 & 4.964 & 0.0129 & \\
Pvalor & 0.0172 & 0.0134 & & \\
\hline
\end{tabular}

OBS: $\mathrm{p}$ valor em parênteses; ${ }^{* *} \mathrm{p}<0.01$.

Fonte: Elaboração dos autores.

instrumentos no combate à proliferação do cigarro, desestimulando o seu consumo entre os já fumantes e evitando a iniciação pelos mais jovens. Esta é a posição, por exemplo, da Organi- zação Mundial da Saúde ${ }^{1}$, que coloca o aumento da tributação como um dos seis pilares efetivos no controle da epidemia do tabaco (MPOWER) e considera que esta medida é a mais efetiva das 
Tabela 3. Prevalência do consumo de cigarros, homens.

\begin{tabular}{lcccc}
\hline \multicolumn{1}{c}{ Variável } & Modelo 1 & Modelo 2 & Modelo 3 & Modelo 4 \\
\hline PUS & 0.00728 & 0.00459 & 0.00510 & 0.0109 \\
& $(0.750)$ & $(0.835)$ & $(0.812)$ & $(0.554)$ \\
TAX & $0.381^{* *}$ & $0.405^{* *}$ & $0.400^{* *}$ & $0.411^{* *}$ \\
& $(0.007)$ & $(0.003)$ & $(0.002)$ & $(0.001)$ \\
lnPIB & -0.0444 & -0.0336 & -0.0376 & -0.0440 \\
& $(0.515)$ & $(0.605)$ & $(0.533)$ & $(0.452)$ \\
AffPIBM & 0.0954 & 0.102 & 0.108 & 0.113 \\
& $(0.195)$ & $(0.156)$ & $(0.100)$ & $(0.076)$ \\
lnSM & -0.0392 & -0.0419 & -0.0411 & -0.0376 \\
& $(0.168)$ & $(0.131)$ & $(0.125)$ & $-141)$ \\
URB & -0.185 & -0.185 & -0.188 & -0.189 \\
& $(0.278)$ & $(0.271)$ & $(0.252)$ & - \\
OECD & 0.0479 & 0.0462 & 0.0459 & - \\
& $(0.579)$ & $(0.586)$ & $(0.581)$ & - \\
MUS & 0.0334 & -0.0115 & - & - \\
ALFH & $(0.621)$ & $(0.837)$ & - & -0.0486 \\
& 0.149 & & & $(0.845)$ \\
cons & $(0.546)$ & -0.00974 & -0.0196 & 31 \\
& -0.0142 & $(0.971)$ & $(0.939)$ & 0.502 \\
N & $(0.958)$ & 31 & 31 & 4.037 \\
R2 & 31 & 0.510 & 0.509 & 0.0445 \\
F & 0.519 & 2.862 & 3.406 & \\
Pvalor & 2.513 & 0.0641 & 0.0505 & \\
\hline
\end{tabular}

OBS: $\mathrm{p}$ valor em parênteses; ${ }^{* *} \mathrm{p}<0.01$.

Fonte: Elaboração dos autores.

estratégias de redução do consumo. O uso intensivo do aumento de tributos é uma das principais estratégias nos países desenvolvidos para o controle da prevalência ${ }^{13}$.

Todas as demais variáveis não foram significantes, inclusive preços e renda, as variáveis que indicam se o país é muçulmano e que mede a capacidade de compra de cigarros, bem como urbanização e taxa de alfabetização. Assim o fato de o país ser mais rico ou mais pobre e se o preço do cigarro é mais alto ou mais baixo, parece não influenciar a prevalência de cigarros. $\mathrm{O}$ mesmo vale se o país tem taxas de alfabetização maiores ou menores, bem como em relação à taxa de urbanização. É um comportamento típico de um vício, como colocado muitas vezes pelas autoridades de saúde.

Porém, quando se estuda a prevalência entre os sexos aparece um comportamento discrepante do obtido para a população total. Em relação aos homens, como mostra a tabela abaixo, os resultados são muito similares ao da população total, com pequena influência de variáveis como renda e preço dos cigarros na decisão de fumar. Entretanto, para as mulheres, outros fatores que vão além da tributação parecem afetar a decisão de fumar, o que pode indicar algumas formas de atuação para as autoridades no sentido de se evitar que o consumo de cigarros se espalhe também entre as adultas.

Assim como para a população total, apenas a tributação está relacionada com a prevalência entre os homens. O argumento da causalidade reversa se aplica aqui também - seguindo recomendações da OMS para combater a alta prevalência de cigarros na população, os países adotaram a tributação como uma das formas de combate ao problema. Por outro lado, nem preço e nem a renda parece afetar o consumo de cigarros pelos homens.

A Tabela 4 apresenta os resultados da regressão para a prevalência entre as mulheres.

Novamente a tributação aparece como variável significativa, agora na prevalência entre as mulheres. A lógica da causalidade inversa permanece válida. 
Tabela 4. Prevalência do consumo de cigarros, mulheres.

\begin{tabular}{|c|c|c|c|c|}
\hline Variável & Modelo 1 & Modelo 2 & Modelo 3 & Modelo 4 \\
\hline PUS & $\begin{array}{r}-0.0394^{*} \\
(0.028)\end{array}$ & $\begin{array}{r}-0.0397^{*} \\
(0.011)\end{array}$ & $\begin{array}{r}-0.0398^{*} \\
(0.010)\end{array}$ & $\begin{array}{r}-0.0405^{* *} \\
(0.008)\end{array}$ \\
\hline TAX & $\begin{array}{c}0.225^{*} \\
(0.030)\end{array}$ & $\begin{array}{c}0.207^{\star} \\
(0.031)\end{array}$ & $\begin{array}{r}0.210^{*} \\
(0.027)\end{array}$ & $\begin{array}{c}0.230^{* *} \\
(0.008)\end{array}$ \\
\hline $\ln \mathrm{PIB}$ & $\begin{array}{r}0.104 \\
(0.058)\end{array}$ & $\begin{array}{r}0.0778 \\
(0.089)\end{array}$ & $\begin{array}{r}0.0800 \\
(0.076)\end{array}$ & $\begin{array}{c}0.0841 \\
(0.056)\end{array}$ \\
\hline AffPIBM & $\begin{array}{r}-0.130 \\
(0.051)\end{array}$ & $\begin{array}{l}-0.0587 \\
(0.278)\end{array}$ & $\begin{array}{r}-0.0713 \\
(0.156)\end{array}$ & $\begin{array}{r}-0.0663 \\
(0.172)\end{array}$ \\
\hline $\operatorname{lnSM}$ & $\begin{array}{r}-0.00565 \\
(0.794)\end{array}$ & - & - & - \\
\hline URB & $\begin{array}{r}0.200 \\
(0.131)\end{array}$ & $\begin{array}{r}0.123 \\
(0.298)\end{array}$ & $\begin{array}{r}0.125 \\
(0.284)\end{array}$ & $\begin{array}{r}0.124 \\
(0.281)\end{array}$ \\
\hline OECD & $\begin{array}{r}0.127^{*} \\
(0.049)\end{array}$ & $\begin{array}{l}0.141^{* *} \\
(0.008)\end{array}$ & $\begin{array}{l}0.144^{* *} \\
(0.006)\end{array}$ & $\begin{array}{l}0.140^{* *} \\
(0.006)\end{array}$ \\
\hline MUS & $\begin{array}{l}0.0156 \\
(0.762)\end{array}$ & $\begin{array}{l}-0.0304 \\
(0.515)\end{array}$ & - & - \\
\hline ALFH & $\begin{array}{r}0.0683 \\
(0.652)\end{array}$ & $\begin{array}{r}0.00399 \\
(0.978)\end{array}$ & $\begin{array}{r}0.0601 \\
(0.600)\end{array}$ & - \\
\hline _cons & $\begin{array}{c}0.0912 \\
(0.684)\end{array}$ & $\begin{array}{l}-0.177 \\
(0.320)\end{array}$ & $\begin{array}{r}-0.151 \\
(0.378)\end{array}$ & $\begin{array}{r}-0.186 \\
(0.233)\end{array}$ \\
\hline $\mathrm{N}$ & 29 & 35 & 35 & 35 \\
\hline R2 & 0.735 & 0.695 & 0.690 & 0.687 \\
\hline $\mathrm{F}$ & 5.855 & 7.409 & 8.585 & 10.23 \\
\hline Pvalor & 0.00503 & 0.00313 & 0.00341 & 0.00396 \\
\hline
\end{tabular}

OBS: $\mathrm{p}$ valor em parênteses; ${ }^{*} \mathrm{p}<0.05,{ }^{* *} \mathrm{p}<0.01$.

Fonte: Elaboração dos autores.

Porém, para o grupo das mulheres, duas outras variáveis são importantes para explicar o comportamento delas em relação ao cigarro. A primeira sugere que mulheres que vivem em nações ricas, dado pela variável dummy OECD, fumam mais do que as que vivem nos demais países. Não é um resultado surpreendente, haja vista os resultados do Indicador de Diferenças entre os sexos (Global Gender Gap Index) do Fórum Econômico Mundial ${ }^{14}$, que aponta de forma consistente que as mulheres nos países desenvolvidos apresentam números bem superiores ao das nações em desenvolvimento. Este indicador examina as diferenças de tratamento entre homens e mulheres em quatro categorias: participação econômica e oportunidades, acesso à educação, saúde e sobrevivência e participação política.

Outra variável importante para as mulheres é o preço do maço de cigarros. Este grupo é especialmente sensível a preço. Preços mais elevados reduzem o consumo de cigarro das mulheres. Ainda que estas tenham mais independência em países desenvolvidos, de forma geral, elas têm me- nos acesso ao emprego formal e recebem salários substancialmente mais baixos do que os homens. Por exemplo, o diferencial de salários entre homens e mulheres em países da OECD é de 17,3\%, enquanto que esta diferença chega a 35\% na África Subsaariana e a $48 \%$ no sudeste asiático ${ }^{15}$.

Ademais, as mulheres estão mais restritas pelas necessidades básicas familiares, principalmente em relação aos filhos, fazendo com que o preço do cigarro acabe se tornando uma barreira, principalmente quando considerada as demais despesas familiares.

\section{Conclusão}

Este artigo procurou analisar os determinantes da prevalência de cigarros considerando um conjunto de 37 países. A análise foi realizada considerando-se não só a população total, mas também, de forma separada, a prevalência entre homens e mulheres. Para tanto, foi feito um exercício econométrico considerando-se como independentes 
um grupo de variáveis econômicas, como preço do maço de cigarros, alíquotas tributárias, renda média, salário mínimo e capacidade de compra de cigarros. Também foram adicionadas variáveis de controle como se o país faz parte da OECD ou se é muçulmano, bem como a sua taxa de alfabetização e urbanização.

Verificou-se que a tributação tem sido o principal mecanismo utilizado pelos países no combate ao consumo de cigarros. Em todos os modelos considerados, e tanto para a população masculina como a feminina, a tributação foi a única variável significativamente correlacionada com a prevalência.

Para os homens e a população total apenas a tributação apresentou-se como significativa. Porém, para as mulheres, outras variáveis são também importantes na explicação da decisão delas de consumir cigarros: a variável dummy OECD e o preço do maço de cigarros. A primeira variável reflete a maior independência cultural, econômica e social que as mulheres desfrutam nos países mais desenvolvidos e a segunda indica que as mulheres, com menos acesso ao emprego formal, recebendo salários substancialmente mais baixos do que os homens e com preocupações familiares básicas, acabam se tornando mais sensíveis ao preço dos cigarros.

Uma limitação relevante do estudo é que se trabalhou apenas com preços e impostos da marca mais vendida em cada país. Com isso, os cigarros ilegais ou contrabandeados, com preços mais baixos e que não pagam tributo, não foram considerados, e podem afetar os resultados, diminuindo o impacto do preço como variável explicativa da prevalência em adultos. Preços mais altos podem não influenciar a prevalência, uma vez que os consumidores migram para cigarros contrabandeados, especialmente em países em desenvolvimento ${ }^{16,17}$

Portanto, apesar da limitação mencionada, as evidências apresentadas neste estudo reforçam a percepção de que a tributação é de fato um instrumento fundamental no controle do consumo de cigarros, mas que no caso específico das mulheres, preços mais elevados e a promoção de maior igualdade com os homens, são também importantes na prevenção do uso do tabaco. 


\section{Referências}

1. Organização Mundial da Saúde (OMS). Report on the global tobacco epidemy. Geneva: OMS; 2010.

2. Eriksen M, Mackay J, Ross H. The Tobacco Atlas. $4^{\text {th }}$ Edition. New York: American Cancer Society and World Lung Foundation; 2012.

3. Instituto Nacional de Alimentação e Nutrição (INAN). Pesquisa nacional sobre saúde e nutrição- PNSN, 1989. Brasília: Ministério da Saúde; 1990.

4. Monteiro CA, Cavalcante T, Moura E, Claro R, Carlos $\mathrm{S}$. Population-based evidence of a strong decline in the prevalence of smokers in Brazil (1989-2003). Bull World Health Organ 2007; 85(7):527-534.

5. Instituto Brasileiro de Geografia e Estatística (IBGE). Pesquisa nacional por amostra de domicílios, 2008. Rio de Janeiro: IBGE; 2009.

6. Aliança de Controle ao Tabagismo - Brasil (ACTBr). Porque aumentar a carga tributária e o preço dos cigarros no Brasil. [acessado 2014 jan 25]. Disponível em: http:// www.actbr.org.br/uploads/conteudo/643_Porque_ aumentar_precos_e_impostos_factsheet.pdf.

7. Gilmore A, Pomerleau J, Mckee M, Rose R, Haerpfer CW, Rotman D, Tumanov S. Prevalence of smoking in 8 countries of the former Soviet Union: results from the living conditions, lifestyles and health study. Am J Public Health 2004; 94(12):2177-2187.

8. Pomerleau J, Gilmore A, Mckee M, Rose R, Haerpfer CW. Determinants of smoking in eight countries of the former Soviet Union: results from the Living Conditions, Lifestyles and Health Study. Addiction 2004; 99(12):1577-1585.

9. Fletcher J. Why have tobacco control policies stalled? Using genetic moderation to examine policy impacts. PLoS ONE 2012: 7(12):e50576. doi:10.1371/journal. pone.0050576.

10. Lugo A, Gallus S, Edefonti V, La Vecchia C. Smoking prevalence and illicit cigarettes trade in 18 European countries. [acessado 2013 nov 10]. Disponível em: http://air.unimi.it/bitstream/2434/222364/2/Lugo_ Abstract_SIB2013.pdf
11. Heston A, Summers R, Aten B. Penn World Table Version 7.1. Philadelphia: Center for International Comparisons of Production, Income and Prices at the University of Pennsylvania; 2012.

12. Pew Research Center. The Future of the Global Muslim Population - projections for 2010-2030. Washington: Pew Research Center; 2011.

13. Molarius A, Parsons RW, Dobson AJ, Evans A, Fortmann S, Jamrozik K, Kuulasmaa K, Moltchanov V, Sans S, Tuomilehto J, Puska P. Trends in cigarette smoking in 36 populations from the early 1980s to the mid-1990s: findings from the WHO MONICA Project. Am J Public Health 2001; 91(1):206-212.

14. World Economic Forum (WEF). The global gender gap report, 2013. Geneva: WEF; 2013.

15. Nopo H, Daza N, Ramos J. Gender earnings gaps in the world. Bonn: IZA; 2011. Discussion Paper no 5736

16. Chaloupka FJ, Peck R, Tauras J, Xu X, Yurekli A. Cigarette excise taxation: the impact of tax structure on prices, revenues, and cigarette smoking. Cambridge: NBER; 2010. Working Paper no 16287

17. Martire K, Mattick R, Doran C, Hall W. Cigarette tax and public health: what are the implications of financially stressed smokers for the effects of price increases on smoking prevalence? Addiction 2011; 106(3):622630.

Artigo apresentado em 17/06/2014

Aprovado em 14/05/2015

Versão final apresentada em 16/05/2015 